\title{
Fast oscillatory activity in the anterior cingulate cortex: dopaminergic modulation and effect of perineuronal net
} loss

\author{
Pascal Steullet *, Jan-Harry Cabungcal, Michel Cuénod and Kim Q. Do \\ Department of Psychiatry, Center of Psychiatric Neuroscience, Centre Hospitalier Universitaire Vaudois and University of Lausanne, Prilly-Lausanne, Switzerland
}

\section{Edited by:}

Laura Cancedda, Istituto Italiano di Tecnologia (IIT), Italy

Reviewed by:

Mark Bevan, Northwestern

University, USA

Richard Deth, Northeastern

University, USA

${ }^{*}$ Correspondence:

Pascal Steullet, Department of

Psychiatry, Center of Psychiatric Neuroscience, Centre Hospitalier Universitaire Vaudois and University of Lausanne, Site de Cery, 1008

Prilly-Lausanne, Switzerland e-mail: pascal.steullet@chuv.ch
Dopamine release in the prefrontal cortex plays a critical role in cognitive function such as working memory, attention and planning. Dopamine exerts complex modulation on excitability of pyramidal neurons and interneurons, and regulates excitatory and inhibitory synaptic transmission. Because of the complexity of this modulation, it is difficult to fully comprehend the effect of dopamine on neuronal network activity. In this study, we investigated the effect of dopamine on local high-frequency oscillatory neuronal activity (in $\beta$ band) in slices of the mouse anterior cingulate cortex (ACC). We found that dopamine enhanced the power of these oscillations induced by kainate and carbachol, but did not affect their peak frequency. Activation of D2R and in a lesser degree D1R increased the oscillation power, while activation of D4R had no effect. These high-frequency oscillations in the ACC relied on both phasic inhibitory and excitatory transmission and functional gap junctions. Thus, dopamine released in the ACC promotes high-frequency synchronized local cortical activity which is known to favor information transfer, fast selection and binding of distributed neuronal responses. Finally, the power of these oscillations was significantly enhanced after degradation of the perineuronal nets (PNNs) enwrapping most parvalbumin interneurons. This study provides new insights for a better understanding of the abnormal prefrontal gamma activity in schizophrenia (SZ) patients who display prefrontal anomalies of both the dopaminergic system and the PNNs.

Keywords: anterior cingulate cortex, dopamine receptors, beta oscillations, perineuronal nets, parvalbumin interneurons, mouse

\section{INTRODUCTION}

Dopamine released in the prefrontal cortex plays a critical role in cognitive function such as working memory, attention and decision making (Seamans and Yang, 2004; Tritsch and Sabatini, 2012). D2R-binding PET studies suggest that dopamine is released in the prefrontal cortex during attention set-shifting (Ko et al., 2009), sustained attention and working memory task (Aalto et al., 2005), and during mild psychological stress (Lataster et al., 2011). Microinjection of dopamine receptor antagonists/agonists in rodent medial prefrontal cortex has revealed that D1R and D4R play a role in working memory (Zhang et al., 2004; Vijayraghavan et al., 2007), D1R and D2R regulate risk-based decision making (St Onge et al., 2011) and are necessary for attention set-shifting responses (Floresco et al., 2006), D4R and D1R differentially modulate encoding of salient and non-salient emotional information (Lauzon et al., 2009). Dopamine is therefore critical for proper processing of information within the prefrontal cortex during a number of cognitive tasks.

Through multiple types of receptors, dopamine exerts complex modulations on the excitability of pyramidal neurons and interneurons. It also regulates excitatory and inhibitory synaptic transmission at either pre- or postsynaptic loci. Moreover, the effect of dopamine varies among cell types, synapses, cortical layers and may depend on the level of neuronal activity (Seamans and Yang, 2004; Tritsch and Sabatini, 2012). Dopamine receptor activation in the prefrontal cortex can modify synaptic inputs to the cortical network (Gurden et al., 2000) and the local recurrent excitatory synapses (Onn et al., 2006). It can also selectively influence the strength of specific outputs to subcortical structures (Gee et al., 2012), increase the inputoutput responses in pyramidal neurons (Thurley et al., 2008), modulate persistent synaptic activity and enhance the signalto-noise ratio (Kroener et al., 2009). Furthermore, the concentration of dopamine associated with its tonic or phasic release determines how this neuromodulator influences information processing via a predominant activation of either D1-or D2type receptors. Seamans and Yang (2004) proposed a model in which multiple inputs in prefrontal cortex would access to the working memory buffers allowing multiple representations when D2R activation is predominant. By contrast, when D1R activation prevails, only strong inputs would produce active and stable network representations. However, due to the complexity of dopamine modulation, it is difficult to fully comprehend the effect of dopamine on prefrontal network activity. Because 
dopamine in the prefrontal cortex modulates the temporal dynamics of feed-forward inhibition (Tierney et al., 2008) and increases the excitability of fast-spiking interneurons (Gorelova et al., 2002; Tseng and O'Donnell, 2007), this neuromodulator could potentially modulate fast rhythmic synchronized neuronal activity, which occurs during many cognitive processes (Howard et al., 2003; Fan et al., 2007; Engell and McCarthy, 2010) favoring information transfer (Sohal et al., 2009), fast selection and binding of distributed neuronal responses (Fries et al., 2007). Local dopaminergic modulation of prefrontal $\gamma$ oscillations has been proposed (Whittington et al., 2011; Furth et al., 2013), but experimental evidence for such a regulation is lacking.

In the present study, we investigated whether dopamine can modulate local fast rhythmic neuronal synchronization in slices of the anterior cingulate cortex (ACC), a region of the medial prefrontal cortex richly innervated by dopaminergic neurons (Descarries et al., 1987; Rivera et al., 2008) and affected in several psychiatric conditions (Fountoulakis et al., 2008; Fornito et al., 2009; Minzenberg et al., 2009; Chan et al., 2011; Frodl and Skokauskas, 2012). The ACC contributes to decision making and conflict monitoring (Botvinick, 2007), cost benefit analysis (Assadi et al., 2009) and empathy (Bernhardt and Singer, 2012). To date, the dopaminergic system in the ACC has been implicated in cost-based decision making (Schweimer and Hauber, 2006), tasks requiring sustained attention and working memory (Aalto et al., 2005) and attention set-shifting (Lumme et al., 2007; Ko et al., 2009). One of the functions of dopamine in the ACC might therefore be the modulation of high-frequency neuronal synchronization to control information processing during some of the above cognitive tasks. If so, an abnormal prefrontal dopaminergic system might contribute to the abnormal $\gamma$ oscillations observed in patients with schizophrenia (SZ). Finally because there is a marked deficit of perineuronal nets (PNNs, specialized extracellular matrix enwrapping most parvalbumin-expressing fast-spiking interneurons which support high-frequency oscillations) in the prefrontal cortex of SZ patients (Mauney et al., 2013), we also examined the effect of PNN loss on fast oscillatory neuronal activity in the ACC.

\section{METHODS \\ ANIMALS}

Experiments were performed on adult ( $\sim 3-5$-month-old) $\mathrm{C} 57 \mathrm{Bl} / 6 \mathrm{~J}$ mice and were approved by the Swiss Veterinary Office of the Canton de Vaud (Switzerland).

\section{Surgery and chondroitinase $A B C$ treatment}

This experiment was designed to assess the effect of PNN removal on fast oscillatory activity in the ACC of adult mice. To do that, PNNs were enzymatically degraded in the ACC via an intracortical injection of chondroitinase $\mathrm{ABC}$ (ChABC, from Proteus vulgaris, Sigma-Aldrich, Switzerland). Mice were anesthetized with ketamine/xylasine $(73 / 11.6 \mathrm{mg} / \mathrm{kg}$, i.p.). Isoflurane was used to maintain the mice in a deep state of anesthesia throughout the surgical procedure. Bilateral craniotomy was performed (Bregma $\sim 1.2$, lateral $\sim 0.25$, depth $\sim 1.25 \mathrm{~mm})$ to inject $1 \mu \mathrm{l} \operatorname{ChABC}(50 \mathrm{U} / \mathrm{ml} ; 0.1 \mu \mathrm{l} / \mathrm{min})$ into one ACC and $1 \mu$ l vehicle solution PBS with $0.1 \%$ BSA) into the contralateral ACC. As analgesics, lidocaine (Wacker Chemie AG, Switzerland) was locally applied while buprenorphine (Temgesic, Essex Chemie AG, Switzerland) was injected $(0.1 \mathrm{mg} / \mathrm{kg}$, s.c. $)$ during surgery. Mice were sacrificed 3 days postinjection for electrophysiological and subsequent morphological assessment.

\section{Electrophysiology}

Anesthetized mice were perfused with oxygenated sucrosecontaining artificial cerebrospinal fluid (ACF) (in mM: 252 sucrose, $3 \mathrm{KCl}, 2 \mathrm{MgSO}_{4}, 1.2 \mathrm{CaCl}_{2}, 1.2 \mathrm{NaH}_{2} \mathrm{PO}_{4}, 24 \mathrm{NaHCO}_{3}$, 10 glucose; $\mathrm{pH} 7.4$ ) for $10 \mathrm{~min}$ prior to decapitation. Paracoronal slices (400 $\mu \mathrm{m}$ thick, Bregma $\sim 1.4-0.6$ ) containing the ACC were prepared with a vibroslicer in cold oxygenated sucrosecontaining ACF, transferred into a "Haas" type interface chamber (kindly provided by MA Whittington) and superfused with oxygenated normal ACF (in mM: $126 \mathrm{NaCl}, 3 \mathrm{KCl}, 1 \mathrm{MgCl}_{2}$, $1.2 \mathrm{CaCl}_{2}, 1.2 \mathrm{NaH}_{2} \mathrm{PO}_{4}, 24 \mathrm{NaHCO}_{3}, 10$ glucose; $\mathrm{pH} 7.4$ ). ACF temperature was slowly raised from room temperature to $\sim 32^{\circ} \mathrm{C}$. Electrophysiological recordings were performed at least 90 min after slicing. Field potentials were recorded with ACFfilled glass electrodes $(\sim 1 \mathrm{M} \Omega)$. Signals were band pass-filtered at $0.3-3000 \mathrm{~Hz}$ and digitized at $5 \mathrm{kHz}$. Oscillatory neuronal activity was generated with a mixture of kainate $(0.8 \mu \mathrm{M})$ and carbachol, $(50 \mu \mathrm{M})$ in $5 \mathrm{mM} \mathrm{KCl-containing} \mathrm{ACF}$. The recording electrode was positioned in the superficial part of layer 5 where the oscillatory activity was most powerful. Typically, a 10-15-min superfusion with kainate + carbachol was necessary to observe stable high-frequency oscillatory activity in the $\beta$ band. This fast oscillatory neuronal activity vanished after removal of carbachol + kainate, but could be induced again to similar levels with these pharmacological agonists. Power spectrum analyses were performed on 60-s recordings using the Welch method (IgorPro6 WaveMetrics, Portland, OR, USA). The power density of fast oscillatory activity was calculated within the $\beta$ band (13-28 Hz). Dopaminergic modulation of this rhythmic neuronal activity was assessed by comparing within the same ACC slice the power and peak frequency of the oscillations induced by carbachol + kainate with those generated by carbachol + kainate in the presence of dopaminergic agonist/antagonist. The concentration of the agonist of a given dopamine receptor type was chosen on the basis of its published constant Ki values for each type of dopaminergic receptors, so the concentration used (0.4-1 $\mu \mathrm{M})$ would activate most of its specific receptors without exciting a large proportion of other dopaminergic receptor types.

We also investigated the contribution of gap junctions, GABAergic and glutamatergic receptors on the generation and maintenance of these local fast neuronal oscillations induced by carbachol + kainate and modulated by dopaminergic agonists. To do so, pharmacological blockers (carbenoxolone, picrotoxin, SYM2206, AP-5) were added to the superfusion after robust and stable oscillations were induced with quinpirole + kainate + carbachol. The power and peak frequency of the oscillations before and after adjunction of these pharmacological blockers were compared. 
To study the effect of PNN loss on oscillations, we recorded and analyzed oscillations induced by co-application of quinpirole, kainate and carbachol in the ACC that were previously intracortically injected with $\mathrm{ChABC}$ and in the corresponding contralateral vehicle-injected ACC (sham). Recordings were performed bilaterally in 3-4 slices at and contiguous to the injection sites. After the recordings, each slice (400 $\mu \mathrm{m}$ thick) was then fixed in $4 \%$ paraformaldehyde and re-cut into $40 \mu \mathrm{m}$ frozen sections for immunolabeling. Two non-contiguous sections of $400 \mu \mathrm{m}$ slice were processed for immunofluorescence to check for qualitative density of PNN which was labeled with the lectin Wisteria Floribunda Agglutinin (WFA). Physiological data from slices displaying no or reduced WFA labeling in the ChABC-injected ACC compared to the contralateral sham ACC were analyzed together. Data from slices displaying no difference of WFA labeling in the ChABC-injected and vehicleinjected sides (slices posterior or anterior to the injection site) were also analyzed separately and used as an additional control dataset.

\section{Immunofluorescence}

Brain sections containing the ACC were first incubated with PBS + Triton $0.3 \%+$ sodium azide $(1 \mathrm{~g} / \mathrm{L})$ containing $2 \%$ normal horse serum, then placed for $48 \mathrm{~h}$ in a solution with a rabbit polyclonal anti-parvalbumin (1:2500; Swant, Switzerland) primary antibody together with the biotin-conjugated lectin Wisteria Floribunda Agglutinin (WFA, 1:2000; Sigma, Switzerland). Sections were then washed, incubated with fluorescent secondary antibody conjugates (goat anti-rabbit IgG (1:300; CY3; Chemicon International, USA) and streptavidin CY2 conjugate (1:300; Millipore Corporation, USA)), and counterstained with 100 ng/ml DAPI (4'-6-diamidino-2-phenylindole; Vector Laboratories Incorporation, California, USA).

\section{Statistical analysis}

Effects of pharmacological agents on oscillations (power and peak frequency) were assessed using paired Student $t$-test (for each recorded ACC slice, comparison between oscillations in absence and presence of the pharmacological agent). When data was not normal based on a Kolmogorov-Smirnov test, Wilcoxon-signed rank test was used. Bonferroni correction was applied when more than two conditions were tested in a same ACC slice and more than one pair comparisons were performed. The effect of PNN degradation by ChABC on oscillations was analyzed using paired Student $t$-test (comparison of oscillations on the ChABC-side and on the contralateral sham side of a same slice).

\section{Chemicals}

Carbachol (carbamylcholine chloride), kainate (kainic acid monohydrate), quinpirole, (+)- sulpiride, (+)-SKF-38393, PD168,077, carbenoxolone disodium salt, picrotoxin were purchased from Sigma (Sigma-Aldrich, Buchs, Switzerland); SYM2206 and L745.870 were from Tocris Bioscience (Bristol, UK); AP-5 (DL-2-amino-5-phosphopentanoic acid) was from Alexis Biochemicals (San Diego, CA); R(+)-SCH23390 HCl was from Research Biochemical International (Sigma-Aldrich, Buchs, Switzerland).

\section{RESULTS \\ ACTIVATION OF DOPAMINE RECEPTORS (D2R AND D1R) INCREASES LOCAL FAST OSCILLATORY ACTIVITY IN THE ACC}

To investigate the potent dopaminergic modulation of fast rhythmic neuronal synchronization, we first searched for a pharmacological method to induce persistent fast neuronal oscillations in slices of the ACC, more precisely in the cg1 area (Franklin and Paxinos, 2008). This region is homologous to the human Brodmann area 24b (Vogt and Paxinos, 2014). A mixture of kainate $(0.8 \mu \mathrm{M})$ and carbachol $(50 \mu \mathrm{M})$ generated small and stable neuronal oscillations in the $\beta$ frequency band, reflecting fast rhythmic neuronal synchronization. We evaluated the effect of dopamine on these oscillations by comparing, within the same slices, the oscillatory activity induced by kainate + carbachol with and without dopamine. Dopamine $(50 \mu \mathrm{M})$ significantly enhanced the power of these oscillations (Figures 1A,B) but did not affect their peak frequencies (mean $\pm \mathrm{SD}, 17.3 \pm 1.3 \mathrm{~Hz}$ vs. $17.2 \pm 1.9 \mathrm{~Hz}$ with dopamine, $n=18$ ).

We then screened for the dopamine receptor type(s) responsible for the modulation of these oscillations. Both the D2R-type agonist, quinpirole $(0.5-1 \mu \mathrm{M})$, and the D1R agonist, SKF-38393 $(0.8 \mu \mathrm{M})$, significantly enhanced the power of these oscillations (Figure 1C) without affecting their peak frequencies (17.3 \pm 1.9 $\mathrm{Hz}$ vs. $16.5 \pm 2.4 \mathrm{~Hz}$ with SKF-38393, $n=21 ; 18.1 \pm 2.1 \mathrm{~Hz}$ vs. $18.6 \pm 2.2 \mathrm{~Hz}$ with quinpirole, $n=30$ ). However, the power enhancement induced by SKF-38393 tended to be weaker than that mediated by quinpirole. Thus, in 8 out of 11 ACC slices where both agonists were tested, the power of oscillations was stronger in the presence of quinpirole than with SKF-38393.

The D2R specific antagonist, sulpiride $(10 \mu \mathrm{M})$, prevented most of the quinpirole-induced enhancement of the oscillations (Figure 2A). In contrast, the D4R antagonist, L745.870 (10 $\mu \mathrm{M})$, did not affect the power of the oscillations induced by quinpirole + kainate + carbachol (Figure 2B), indicating that D2R but not $\mathrm{D} 4 \mathrm{R}$ activation causes enhancement of these high-frequency oscillations. The inefficiency of D4R was further confirmed as the specific D4R agonist, PD168.077 $(0.4 \mu \mathrm{M})$, did not alter neither the power (Figure 2C) nor the frequency of the oscillations $(18.7 \pm 1.9 \mathrm{~Hz}$ vs. $18.8 \pm 2.2$ with $\mathrm{PD} 168.077, n=7)$ induced by kainate + carbachol. The selective D1R antagonist, SCH23390 ( $5 \mu \mathrm{M})$, prevented the power increase induced by SKF-38393 (Figure 3), confirming that D1R activation also enhances these oscillations in the ACC. Taken together, the data demonstrated a role of $\mathrm{D} 2 \mathrm{R}$ and $\mathrm{D} 1 \mathrm{R}$ receptors in the modulation of persistent fast oscillatory activity in the local cortical circuitry of the ACC.

\section{FAST OSCILLATORY ACTIVITY IN THE ACC REOUIRES GABA RECEPTORS, AMPA RECEPTORS AND GAP JUNCTIONS}

We then identified the neurotransmitter systems and receptors required to generate these high-frequency oscillations induced in the ACC by kainate + carbachol and modulated by dopaminergic receptor agonists. Blocking $\mathrm{GABA}_{\mathrm{A}}$ receptors with picrotoxin $(50 \mu \mathrm{M})$ fully disrupted the oscillations generated by quinpirole, kainate and carbachol (Figure 4A) and instead led to epilepticform activity (present in 6 out of 8 slices) (Figure 4A). The selective AMPA receptor antagonist, SYM2206 (25-30 $\mu \mathrm{M})$, also 


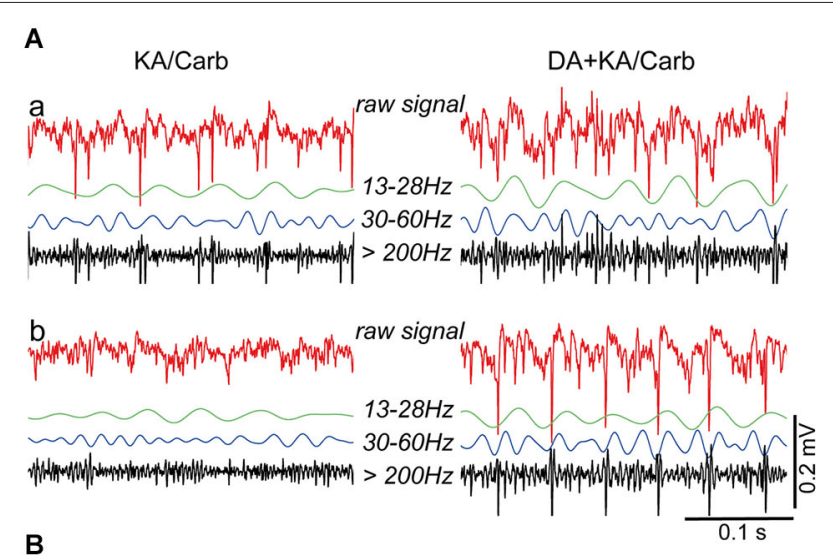

B
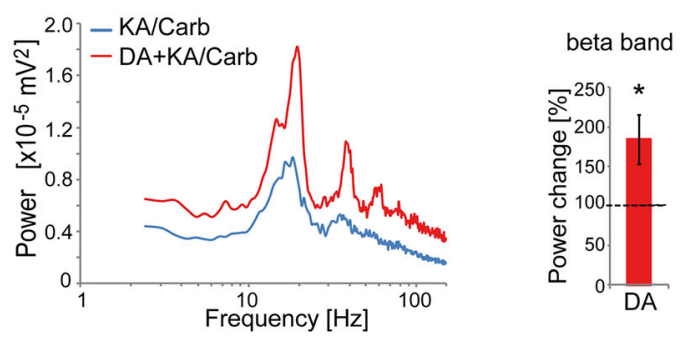

C
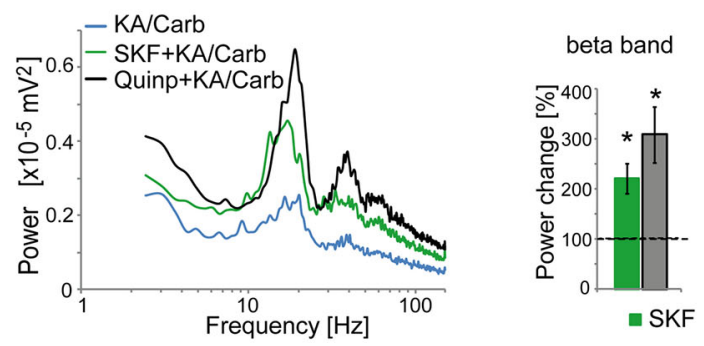

FIGURE 1 | Dopaminergic modulation of high-frequency oscillations in the anterior cingulate cortex (ACC). (A) Illustration of recordings from two ACC slices ( $a$ and b) superfused with kainate + carbachol (KA/Carb) in absence (left) and presence (right) of $50 \mu \mathrm{M}$ dopamine (DA). For each recording, the raw unfiltered signal (red), the filtered signals in the $\beta$ band (13-28 Hz, green), $\gamma$ band (30-60 Hz, blue) and high-frequency band ( $>200$ $\mathrm{Hz}$, black) are displayed. (B) DA significantly enhances the power of high-frequency oscillations (in the $\beta$ band) induced by KA/Carb (two-tailed, $P=0.008, n=8$ ). Left: power spectra of recordings (mean of pooled data). Right: DA-induced change in the power of the oscillations $(100 \%$ corresponds to the power of oscillations elicited by KA/Carb in absence of DA). ${ }^{*} P<0.05$, significantly different from the KA/Carb condition. (C) The D2R agonist (quinpirole, Quinp, $1 \mu \mathrm{M}$ ) and the D1R agonist (SKF-38393, SKF, $0.8 \mu \mathrm{M}$ ) significantly enhance the power of the oscillations induced by KA/Carb (two-tailed, for Quinp: $P=0.003$; for SKF: $P=0.004, n=11$ ). Left: power spectra of recordings (mean of pooled data). Right: Change in the power of the oscillations induced by Quinp and SKF, respectively $(100 \%$ corresponds to the power of oscillations elicited by KA/Carb in absence of DA agonists). ${ }^{*} P<0.05$, significantly different from the KA/Carb condition after Bonferroni correction. Bars, sem.

abolished these oscillations indicating the requirement of AMPA receptor activation (Figure $4 \mathrm{~B}$ ). In contrast, blocking NMDA receptors with AP-5 $(50 \mu \mathrm{M})$ did not alter these oscillations (Figure 4C). Carbenoxolone $(100 \mu \mathrm{M})$, a compound known to impair the function of gap junctions, also disrupted the

\section{A}
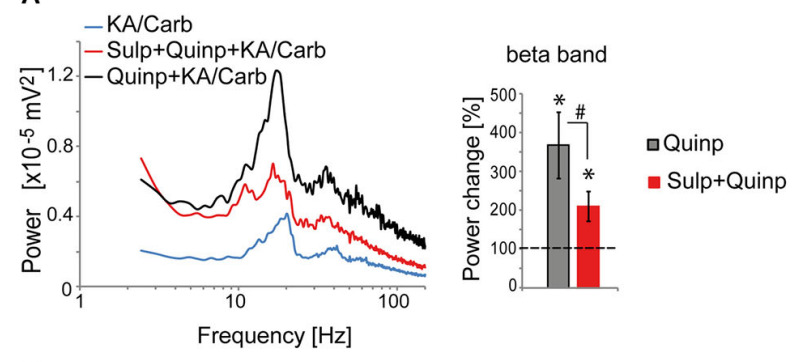

B
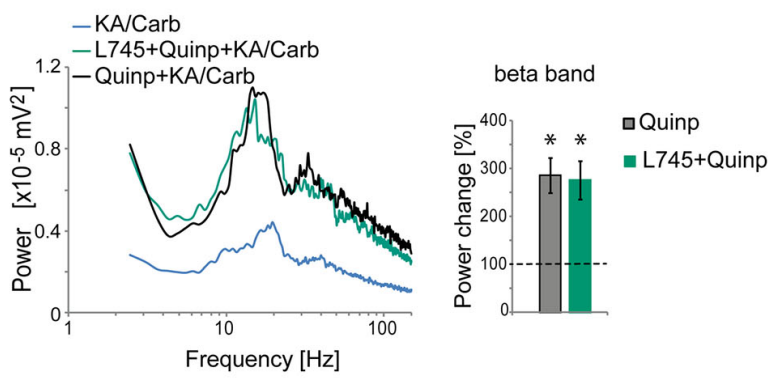

C

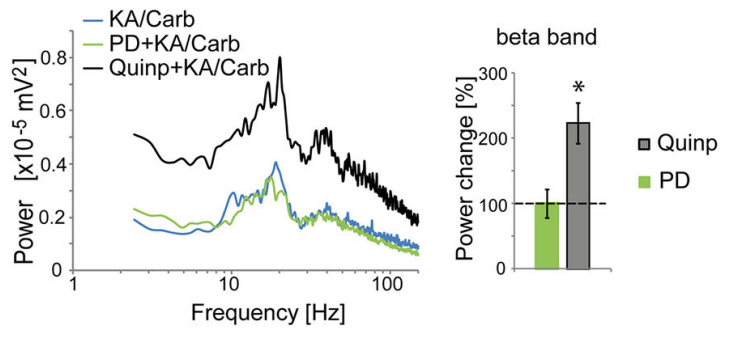

FIGURE 2 | Activation of D2R, but not D4R, enhances the power of high-frequency oscillations in the ACC. (A) Activation of D2R enhances the power of the oscillations induced by kainate + carbachol (KA/Carb) (one-tailed, $P=0.001, n=12$ ). The D2R antagonist (sulpiride, Sulp, $10 \mu \mathrm{M}$ ) significantly reduces the power increase induced by quinpirole (Quinp, $1 \mu \mathrm{M}$ ) (one-tailed, $P=0.001$ ). However, Sulp does not fully prevent the Quinp-induced increase in power (Quinp + Sulp vs. no D2R modulator condition: one-tailed, $P=0.007$ ). Left: power spectra of recordings (mean of pooled data). Right: Change in the power of the oscillations induced by Quinp and by Quinp + Sulp, respectively (100\% corresponds to the power of oscillations elicited by KA/Carb in absence of dopaminergic modulators). ${ }^{*} P<0.05$, significantly different from the KA/Carb condition after Bonferroni correction; ${ }^{\#} P<0.05$, significantly different between the two conditions after Bonferroni correction. (B) The D4R antagonist (L745.870, $L 745,10 \mu \mathrm{M})$ does not decrease the Quinp-induced enhancement of the power of oscillations elicited by KA/Carb (Quinp vs. Quinp + L754: $P>0.05$, $n=15)$. Quinp as well as Quinp + L745 increase significantly the power of oscillations elicited by KA/Carb (for both, one-tailed, $P<0.0005)$. Left: power spectra of recordings (mean of pooled data). Right: Change in the power of the oscillations induced by Quinp and by Quinp $+\mathrm{L} 745$, respectively $(100 \%$ corresponds to the power of oscillations elicited by $\mathrm{KA}$ /Carb in absence of dopaminergic modulators). ${ }^{*} P<0.05$, significantly different from the KA/Carb condition after Bonferroni correction. (C) Activation of D4R does not enhance the power of the oscillations that are induced by KA/Carb. While Quinp significantly increases the power (one-tailed, $P=0.009, n=7$ ), the D4R agonist (PD168.077, PD, $0.4 \mu \mathrm{M}$ ) does not (one-tailed, $P>0.05$ ). Left: power spectra of recordings (mean of pooled data). Right: Change in the power of the oscillations induced by Quinp and PD, respectively (100\% corresponds to the power of oscillations elicited by KA/Carb in absence of DA agonists). ${ }^{*} P<0.05$, significantly different from the KA/Carb condition after Bonferroni correction. Bars, sem. 


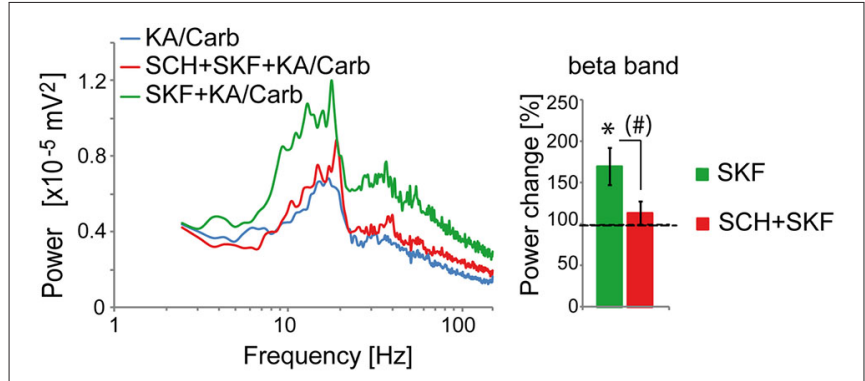

FIGURE 3 | Activation of D1R enhances the power of high-frequency oscillations in the ACC. The enhancement of the power of the oscillations that is induced by the D1R agonist (SKF-38393, SKF, $0.8 \mu \mathrm{M}$ ) (one-tailed, $P=0.0085, n=10)$ is prevented by the D1R antagonist ( $\mathrm{SCH} 23390, \mathrm{SCH}, 5$ $\mu \mathrm{M}$ ). SCH reduces the power increase induced by SKF (one-tailed, $P=$ 0.02 ) and the power is not significantly higher in the SKF + SCH compared to the no D1R modulator condition (one-tailed, $P>0.05$ ). Left: power spectra of recordings (mean of pooled data). Right: Change in the power of the oscillations induced by SKF and by SKF + SCH, respectively (100\% corresponds to the power of oscillations elicited by KA/Carb in absence of dopaminergic modulators). ${ }^{*} P<0.05$, significantly different from the KA/Carb condition after Bonferroni correction; $\# P<0.05$, but no more significantly different between the two conditions after Bonferroni correction. Bars, sem.

oscillations (Figure 4D). Together, these data indicated that this fast oscillatory activity in the ACC is generated within a local network of excitatory and inhibitory neurons requiring functional gap junctions, phasic AMPA receptor-dependent excitation, and $\mathrm{GABA}_{\mathrm{A}}$ receptor-dependent inhibition.

\section{ABSENCE OF INTACT PERINEURONAL NETS ENHANCES THE POWER OF HIGH-FREQUENCY OSCILLATIONS}

Results from a previous study suggest that the fast rhythmic synchronized activity, which can be induced by quinpirole, kainate and carbachol in the ACC, is dependent on parvalbumin interneurons (Cabungcal et al., 2013). Indeed, a deficit in these fast oscillations was associated with a reduced number of parvalbumin-immunoreactive neurons in fully adult $\mathrm{Gclm} \mathrm{KO}$ mice (Cabungcal et al., 2013), which have a limited capacity to produce the antioxidant glutathione (Steullet et al., 2010). Most matured parvalbumin interneurons are enwrapped by a specialized extracellular matrix, the PNNs, which consists of chondroitin sulfate proteoglycans (e.g., versican, aggrecan, neurocan, brevican), hyaluronan, tenascin and link proteins (Kwok et al., 2011). These PNNs promote synaptic and network stability, are involved in the maturation and phenotypic maintenance of parvalbumin interneurons and protect them against oxidative stress (Sugiyama et al., 2009; Kwok et al., 2011; Cabungcal et al., 2013). On this basis, we examined whether PNNs play a role in the maintenance of the oscillatory activity induced by quinpirole, kainate and carbachol. The PNNs were degraded unilaterally in one ACC of adult mice by a local injection of chondroitinase ABC (ChABC), an enzyme that breaks down chondroitin sulfates and hyalorunan. This treatment led to absence of labeling by WFA (Wisteria Floribunda Agglutinin), a lectin that preferentially binds to the PNNs around parvalbumin interneurons (Figure 5A). About 3-days post-injection, mice were sacrificed and electrophysiological recordings were performed on slices containing the
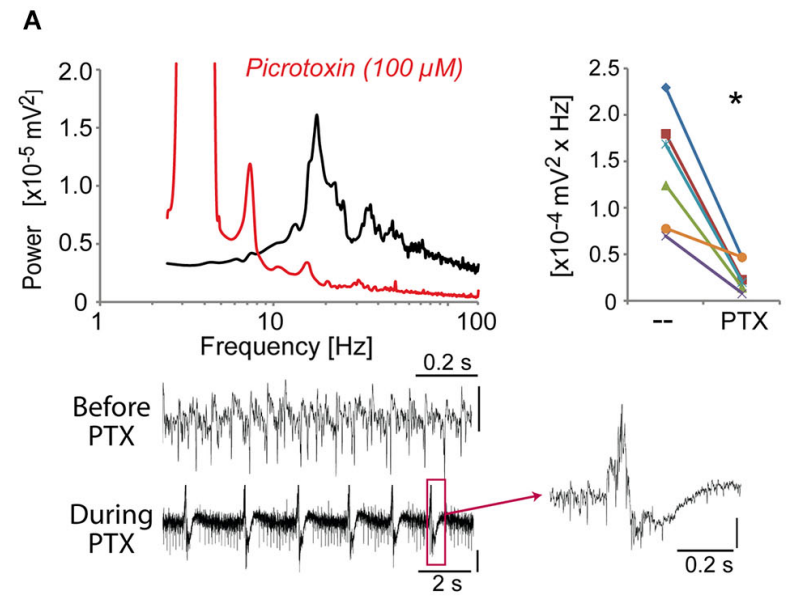

B
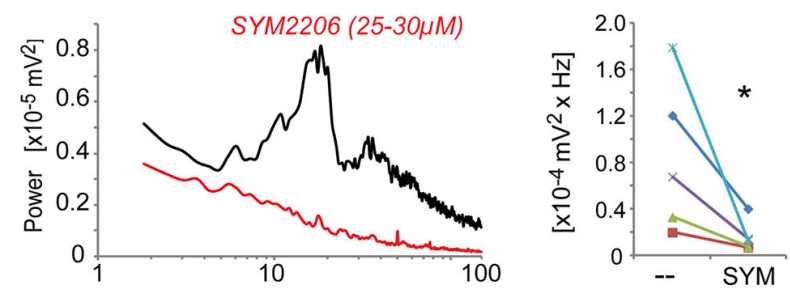

C
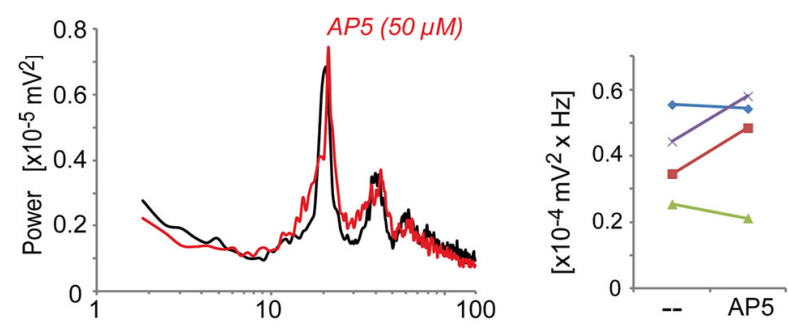

D
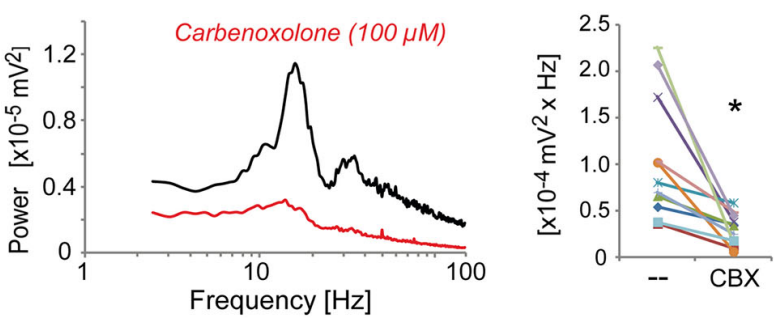

FIGURE 4 | The fast oscillatory activity that is elicited by a mixture of quinpirole, kainate and carbachol (Quinp/KA/Carb) requires activation of $\mathrm{GABA}_{\mathrm{A}}$ receptors, AMPA receptors, and functional gap junctions. (A) Inhibition of $\mathrm{GABA}_{A}$ receptors (with picrotoxin, PTX, $100 \mu \mathrm{M}$, for $30 \mathrm{~min}$ ) abolishes the high-frequency oscillations (one-tailed, $P=0.0025, n=6$ ). Traces show recordings before and during PTX application. Note the regular epilepticform activity following PTX superfusion. The right trace shows one of these epileptic-like events. Vertical scales: $0.1 \mathrm{mV}$. (B) Inhibition of AMPA receptors (with SYM2206, SYM, 25-30 $\mu$ M, for $40 \mathrm{~min}$ ) abolishes the oscillations (one-tailed, $P=0.023, n=5$ ). (C) Inhibition of NMDA receptors (with AP5, $50 \mu \mathrm{M}$, for $40 \mathrm{~min}$ ) does not affect the oscillations (one-tailed, $P>0.05, n=4)$. (D) Functional impairment of gap junctions (with carbenoxolone, CBX, $100 \mu \mathrm{M}$, for $60 \mathrm{~min}$ ) abolishes the oscillations (one-tailed, $P=0.007, n=11$ ). Left graphs: power spectra of recordings

(Continued) 


\section{FIGURE 4 | Continued}

(mean of pooled data) during superfusion with Quinp/KA/Carb in the absence (black) or presence (red) of the drugs. Note for the right graphs: the power values in the $\beta$ band recorded in the same ACC before and after application of the drugs are connected by a line. ${ }^{*} P<0.05$.

ChABC-injected ACC and its contralateral control Sham ACC. The mixture of quinpirole, kainate and carbachol induced highfrequency oscillations in both ChABC-injected and Sham ACC. The power of the oscillations was however significantly higher in the ChABC-injected ACC (which display no WFA labeling as verified post-hoc by immunofluorescence) than in their respective contralateral sham ACC (which show normal WFA labeling) (Figure 5B). By contrast, in slices anterior or posterior to the ChABC injection site where the PNNs remained intact, the power of oscillations was not significantly different between the ACC of both hemispheres (Figure 5C). This indicated that the absence of intact PNNs around parvalbumin interneurons does not disrupt the fast rhythmic activity in the ACC, but rather enhances the power of the oscillations associated with this network activity. The effect of PNN degradation on the power of the oscillations further supports a role for parvalbumin interneurons in this fast oscillatory neuronal activity in the ACC.

\section{DISCUSSION}

In the present study, we show that the power of fast oscillatory activity induced by kainate + carbachol is increased by D2R and $\mathrm{D} 1 \mathrm{R}$ activation in ACC slices of mice. These oscillations require functional AMPA receptors, $\mathrm{GABA}_{\mathrm{A}}$ receptors and gap junctions, and are enhanced in absence of intact PNNs.

The enhancement of local fast oscillatory neuronal activity by dopamine suggests that this neuromodulator improves information transfer (Sohal et al., 2009) and fast selection and binding of distributed neuronal responses (Fries et al., 2007) within the ACC. Interestingly, activation of either D1R or D2R gives rise to a similar modulation of these oscillations. This contrasts with the usual opposite effects of these two types of receptors on pyramidal neuron excitability, GABAergic and glutamatergic synaptic transmission (Tritsch and Sabatini, 2012) and even coupling between neurons (Onn and Grace, 1994). This suggests that dopamineinduced enhancement of high-frequency synchronization is a primordial function in the ACC and may be essentially driven by fast-spiking interneurons whose excitability is increased by the activation of both D1R and D2R receptors (Gorelova et al., 2002; Tseng and O'Donnell, 2007; Towers and Hestrin, 2008). Other data further support a central role of fast-spiking interneurons in the generation of these fast oscillations. Thus, we found a negative correlation between the power of these oscillations and the number of parvalbumin-immunoreactive interneurons in the ACC of Gclm KO mice, an animal model of redox dysregulation and vulnerability for oxidative stress in SZ (Cabungcal et al., 2013). The number of parvalbumin interneurons and the power of these oscillations were not different in young adult $\mathrm{Gclm} \mathrm{KO}$ and WT mice, while in older individuals a decrease in number of parvalbumin-immunoreactive interneurons in $\mathrm{Gclm} \mathrm{KO}$ mice was associated with impaired oscillations. Moreover, our

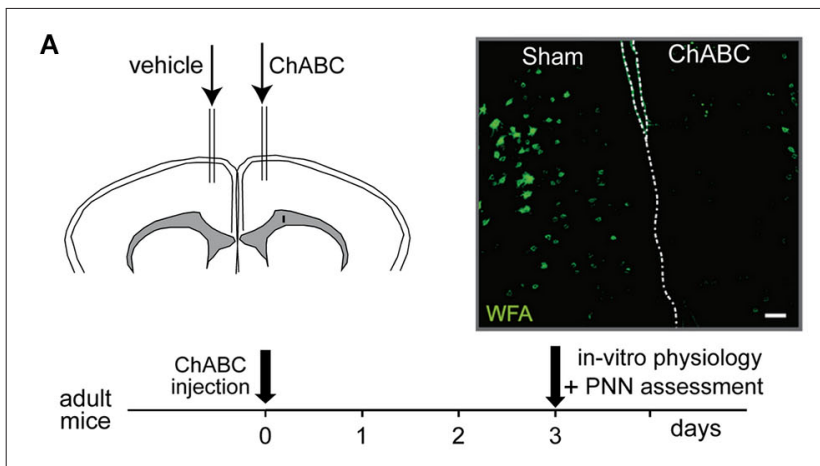

B
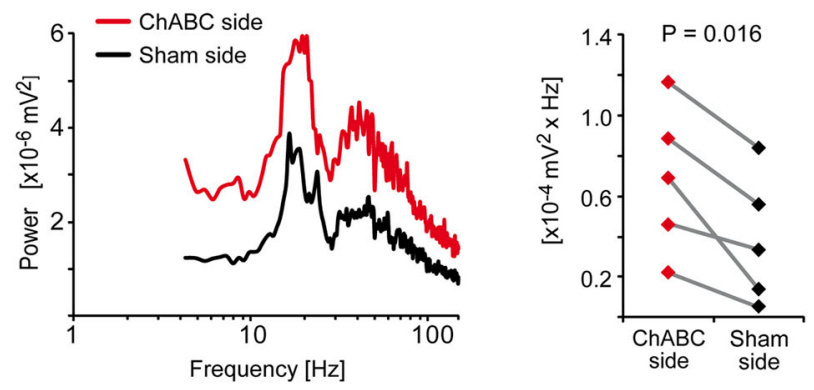

C
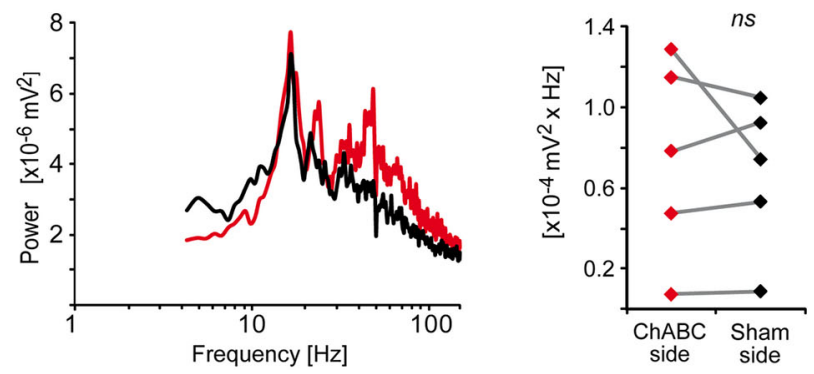

FIGURE 5 | Enzymatic removal of the perineuronal nets (PNNs) by local injection of chondroitinase $A B C$ (ChABC) increases the power of high-frequency oscillations induced by a mixture of quinpirole, kainate and carbachol (Quinp/KA/Carb). (A) Schema of the experimental protocol of $\mathrm{ChABC}$ and control vehicle injections followed by electrophysiological and histological assessment. Micrograph illustrates the integrity of PNNs (as assessed with WFA labeling) in the vehicle-injected side (sham ACC, left) and in the ChABC-injected side (right). Dashed lines indicate separation between hemispheres. Scale: $80 \mu \mathrm{m}$. (B) In coronal slices that show a degradation of PNNs in the ChABC-injected ACC (as assessed by the WFA labeling), the power of the oscillations is significantly larger in the ChABC-injected ACC compared to the contralateral control ACC (sham-injected). (C) In coronal slices that are anterior or posterior to the ChABC injection site (the PNNs remain intact as assessed by WFA labeling), the power of the oscillations in the ACC of the two hemispheres is not significantly different from each other. Left graphs: power spectra of

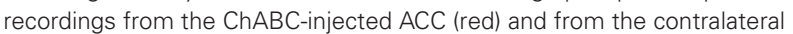
sham ACC (black) (mean of pooled data). Note for the right graphs: the power values in the $\beta$ band recorded in the ChABC-injected side and the control sham side from the same brain slice are connected by a line.

present work shows that enzymatic degradation of the PNNs that enwrap most parvalbumin interneurons also affects these oscillations. Since PNNs are involved in synaptic and network stability (Sugiyama et al., 2009; Kwok et al., 2011), we expected 
that PNN degradation might disrupt fast neuronal synchronization. Instead, we observed an enhanced power of these highfrequency oscillations. These results are however in line with the power increase in $\beta / \gamma$ oscillations observed in mice deficient in tenascin-R, a component and stabilizer of PNNs (Gurevicius et al., 2004). Because PNNs act as a cation buffer via their polyanionic nature, they may slow down or reduce cation exchange through membrane ion channels and therefore limit the interneuron excitability. Indeed, PNN degradation with ChABC lowered the excitability threshold of fast-spiking interneurons (Dityatev et al., 2007 ), which could result in stronger $\beta / \gamma$ oscillations.

While the local fast neuronal synchronization in the ACC critically depends on GABAergic neurons, as blocking $\mathrm{GABA}_{\mathrm{A}}$ receptors fully disturbs high-frequency oscillations, excitatory pyramidal neurons are also necessary. Indeed, blocking AMPA receptors abolishes these oscillations. Thus, the fast oscillations in the ACC that are modulated by dopamine are generated by a local network of excitatory and inhibitory neurons (including parvalbumin interneurons) and require gap junctions and both phasic AMPA receptor-dependent excitation and $\mathrm{GABA}_{\mathrm{A}}$ receptor-dependent inhibition. The type of neuronal network engaged in the persistent high-frequency oscillations in the ACC resembles those found in the auditory cortex (Roopun et al., 2010) and in the superficial layers of the somatosensory cortex (Roopun et al., 2006), but differs from that of the motor cortex (Yamawaki et al., 2008) or the deep layer of the somatosensory cortex (Roopun et al., 2006). Indeed, the pharmacologicallyinduced persistent fast oscillations in the motor cortex did not depend on AMPA receptors and the $\beta$ oscillations in the deep layer of the somatosensory cortex did not require AMPA and $\mathrm{GABA}_{\mathrm{A}}$ receptors.

Dopamine modulation of fast rhythmic neuronal synchronization has been suggested by several authors (Whittington et al., 2011; Furth et al., 2013) and simulated from artificial neuronal networks (Kuznetsova and Deth, 2008; Kömek et al., 2012). Dopamine modulation of $\gamma$ oscillations has been described in the hippocampus (Weiss et al., 2003; Wójtowicz et al., 2009; Andersson et al., 2012). However, the dopaminergic modulation of high-frequency local neuronal synchronization in the neocortex has not been much investigated. DRD4 and DAT1 polymorphisms modulate auditory-evoked $\gamma$ responses in humans, suggesting a dopaminergic modulation of cortical networks (Demiralp et al., 2007). Recently, Kocsis et al. (2013) have shown that administration of a D4R agonist increases $\gamma$ oscillations in hippocampus and several cortical regions (including prefrontal cortex) in awake rats. However, D4R activation did not enhance the high-frequency oscillations induced by kainate + carbachol within a local neuronal network of the ACC. The network of neurons engaged in a high-frequency synchronized activity in slices via pharmacological activation of cholinergic and glutamatergic receptors might be different from the one engaged in-vivo. The cortical activity in-vivo is driven and modulated by the afferents of many brain regions. Therefore, we cannot exclude that the increase of prefrontal $\gamma$ oscillations following injection of a D4R agonist may be primarily due to the action of D4R in other regions, and not to a direct modulation of the local cortical network. Moreover, the direction and the nature (type of receptors involved) of the local dopaminergic modulation of high-frequency oscillations vary across brain regions and even across subregions of the medial prefrontal cortex. Thus, dopamine increases and decreases high-frequency oscillatory activity in the prelimbic and ventral infralimbic cortex, respectively (Glykos et al., 2012). On the other hand, the power of $\gamma$ oscillations in the hippocampus is enhanced by $\mathrm{D} 4 \mathrm{R}$ activation, while D1/5R activation has an opposite effect (Weiss et al., 2003; Wójtowicz et al., 2009; Andersson et al., 2012). The dopamine-induced changes of synaptic and spike activity in ACC pyramidal neurons and interneurons that lead to an increase of fast oscillations remain however undetermined. Since D1R and D2R activation promote the firing of prefrontal fast-spiking interneurons (Gorelova et al., 2002; Tseng and O'Donnell, 2007; Towers and Hestrin, 2008), dopamine might recruit an increasing number of these interneurons and enhance their synchronization as observed in the hippocampus (Andersson et al., 2012). A more in depth study to elucidate how dopamine in the ACC modulates synaptic input integration and the dynamic of spike generation in pyramidal neurons and interneurons during fast oscillatory activity is needed to better understand the dopamine role in information processing within the local ACC neuronal circuit.

The ACC is part of the attention executive network. This prefrontal region links sensory information with rules or expectations to generate motor responses. It is implicated in learning and predicting the likely outcome of actions through evaluation of the probable and actual outcomes of one's action (Alexander and Brown, 2011) or actions from other individuals (Rushworth et al., 2007). The dopaminergic system in the ACC plays a role in costbased decision making (Schweimer and Hauber, 2006), cognitive tasks such as attention set-shifting tasks (Lumme et al., 2007; Ko et al., 2009) and sustained attention and working memory tasks (Aalto et al., 2005). Therefore, it is quite plausible that, in the ACC, dopamine improves the flow of information processing that is associated with local fast neuronal synchrony during tasks requiring attention, working memory and decisions (Howard et al., 2003; Engell and McCarthy, 2010).

In psychiatric diseases such as SZ, the ACC is affected and its activation often abnormal (Dolan et al., 1995; Minzenberg et al., 2009; Kyriakopoulos et al., 2012). Specifically, attention executive function, decision making and cost benefit analysis that all require the ACC are impaired in patients. Interestingly, the dopaminergic system in the ACC is also altered, with increased D1R binding (Abi-Dargham et al., 2012) and decrease D2R binding (Suhara et al., 2002) in drug-naïve SZ compared to healthy subjects. A line of indirect evidence also suggests that SZ is characterized by a hyperfunction and hypofunction of the dopaminergic system in the striatum and the prefrontal cortex, respectively (Simpson et al., 2010). Noteworthy, the failure of a cognitive task to induce an increase in cerebral blood flow in the ACC of SZ patients can be significantly recovered by the dopaminergic agonist, apomorphine (Dolan et al., 1995; Fletcher et al., 1996). Because haemodynamic signals correlate with the power of $\gamma$ oscillations (Niessing et al., 2005; Mulert et al., 2010; Scheeringa et al., 2011), it is therefore possible that abnormal dopaminergic transmission in the ACC and other prefrontal regions contributes to abnormal modulation of induced $\gamma$ oscillations, as observed in patients. 
Basar-Eroglu et al. (2007) found that the power of $\gamma$ oscillations in patients fails to be enhanced during a high-demanding working memory task. Likewise, the induced $\gamma$ oscillations during a high cognitive control tasks were impaired in frontal areas of patients (Cho et al., 2006). By contrast, the power of baseline frontal $\gamma$ oscillations in patients is often higher compared to healthy controls (Basar-Eroglu et al., 2007; Barr et al., 2010; Bandyopadhyaya et al., 2011; Gandal et al., 2012). Intuitively, these strong spontaneous prefrontal $\gamma$ oscillations in patients reveal an apparent contradiction with the anomalies of the network associated with the fast-spiking parvalbumin-expressing interneurons (Lewis et al., 2011). Some data suggest however that a default in NMDAR function in fast-spiking interneurons could contribute to enhanced spontaneous $\gamma$ oscillations in patients. Indeed, a study reports a drastic decrease in number of GAD67-positive neurons expressing the NMDA receptor subunit NR2A in prefrontal cortex of patients (Woo et al., 2008) and a lack of NMDAR neurotransmission in parvalbumin cells via a specific deletion of NR1 on these cells leads to enhanced baseline cortical $\gamma$ rhythms (Carlén et al., 2012). On the other hand, it is also plausible that reduced or abnormal PNNs in prefrontal cortex of patients (Mauney et al., 2013) could contribute to the enhanced baseline frontal $\gamma$ rhythms that are sometimes observed in patients.

To conclude, we have demonstrated a robust dopaminergic modulation of local high-frequency oscillations in the ACC and an enhanced power of these oscillations in absence of intact PNNs. Our data provide new insights on the modulation of highfrequency neuronal synchronization in the prefrontal cortex and may bring a novel perspective for understanding the abnormal $\gamma$ oscillations in the frontal cortex of SZ patients.

\section{AUTHOR CONTRIBUTIONS}

Pascal Steullet, Jan-Harry Cabungcal, and Kim Q. Do designed research; Pascal Steullet performed and analyzed electrophysiological experiments; Jan-Harry Cabungcal performed surgery and immunofluorescence; Pascal Steullet wrote the paper; all authors critically revised the manuscript.

\section{ACKNOWLEDGMENTS}

This work was supported by the Swiss National Science Foundation (\# 310030_135736/1 to Kim Q. Do and Pascal Steullet), "Loterie Romande", Fondation Damm-Etienne, Alamaya Foundation. We thank Adeline Cottier for her technical assistance and Miles A. Whittington for the "Haas" type interface chamber.

\section{REFERENCES}

Aalto, S., Bruck, A., Laine, M., Nagren, K., and Rinne, J. O. (2005). Frontal and temporal dopamine release during working memory and attention tasks in healthy humans: a positron emission tomography study using the high-affinity dopamine D2 receptor ligand [11C]FLB 457. J. Neurosci. 25, 2471-2477. doi: 10. 1523/jneurosci.2097-04.2005

Abi-Dargham, A., Xu, X., Thompson, J. L., Gil, R., Kegeles, L. S., Urban, N., et al. (2012). Increased prefrontal cortical $D(1)$ receptors in drug naive patients with schizophrenia: a PET study with [(1)(1)C]NNC112. J. Psychopharmacol. 26, 794-805. doi: 10.1177/0269881111409265

Alexander, W. H., and Brown, J. W. (2011). Medial prefrontal cortex as an actionoutcome predictor. Nat. Neurosci. 14, 1338-1344. doi: 10.1038/nn.2921

Andersson, R., Johnston, A., and Fisahn, A. (2012). Dopamine D4 receptor activation increases hippocampal gamma oscillations by enhancing synchronization of fast-spiking interneurons. PLoS One 7:e40906. doi: 10.1371/journal.pone. 0040906

Assadi, S. M., Yucel, M., and Pantelis, C. (2009). Dopamine modulates neural networks involved in effort-based decision-making. Neurosci. Biobehav. Rev. 33, 383-393. doi: 10.1016/j.neubiorev.2008.10.010

Bandyopadhyaya, D., Nizamie, S. H., Pradhan, N., and Bandyopadhyaya, A. (2011). Spontaneous gamma coherence as a possible trait marker of schizophrenia-An explorative study. Asian J. Psychiatr. 4, 172-177. doi: 10.1016/j.ajp.2011.06.006

Barr, M. S., Farzan, F., Tran, L. C., Chen, R., Fitzgerald, P. B., and Daskalakis, Z. J. (2010). Evidence for excessive frontal evoked gamma oscillatory activity in schizophrenia during working memory. Schizophr. Res. 121, 146-152. doi: 10. 1016/j.schres.2010.05.023

Basar-Eroglu, C., Brand, A., Hildebrandt, H., Karolina Kedzior, K., Mathes, B., and Schmiedt, C. (2007). Working memory related gamma oscillations in schizophrenia patients. Int. J. Psychophysiol. 64, 39-45. doi: 10.1016/j.ijpsycho. 2006.07.007

Bernhardt, B. C., and Singer, T. (2012). The neural basis of empathy. Annu. Rev. Neurosci. 35, 1-23. doi: 10.1146/annurev-neuro-062111-150536

Botvinick, M. M. (2007). Conflict monitoring and decision making: reconciling two perspectives on anterior cingulate function. Cogn. Affect. Behav. Neurosci. 7, 356-366. doi: 10.3758/cabn.7.4.356

Cabungcal, J. H., Steullet, P., Morishita, H., Kraftsik, R., Cuenod, M., Hensch, T. K., et al. (2013). Perineuronal nets protect fast-spiking interneurons against oxidative stress. Proc. Natl. Acad. Sci. U S A 110, 9130-9135. doi: 10.1073/pnas. 1300454110

Carlén, M., Meletis, K., Siegle, J. H., Cardin, J. A., Futai, K., Vierling-Claassen, D., et al. (2012). A critical role for NMDA receptors in parvalbumin interneurons for gamma rhythm induction and behavior. Mol. Psychiatry 17, 537-548. doi: 10. 1038/mp.2011.31

Chan, R. C., Di, X., McAlonan, G. M., and Gong, Q. Y. (2011). Brain anatomical abnormalities in high-risk individuals, first-episode and chronic schizophrenia: an activation likelihood estimation meta-analysis of illness progression. Schizophr. Bull. 37, 177-188. doi: 10.1093/schbul/sbp073

Cho, R. Y., Konecky, R. O., and Carter, C. S. (2006). Impairments in frontal cortical gamma synchrony and cognitive control in schizophrenia. Proc. Natl. Acad. Sci. U S A 103, 19878-19883. doi: 10.1073/pnas.0609440103

Demiralp, T., Herrmann, C. S., Erdal, M. E., Ergenoglu, T., Keskin, Y. H., Ergen, M., et al. (2007). DRD4 and DAT1 polymorphisms modulate human gamma band responses. Cereb. Cortex 17, 1007-1019. doi: 10.1093/cercor/bhl011

Descarries, L., Lemay, B., Doucet, G., and Berger, B. (1987). Regional and laminar density of the dopamine innervation in adult rat cerebral cortex. Neuroscience 21, 807-824. doi: 10.1016/0306-4522(87)90038-8

Dityatev, A., Bruckner, G., Dityateva, G., Grosche, J., Kleene, R., and Schachner, M. (2007). Activity-dependent formation and functions of chondroitin sulfate-rich extracellular matrix of perineuronal nets. Dev. Neurobiol. 67, 570-588. doi: 10. 1002/dneu.20361

Dolan, R. J., Fletcher, P., Frith, C. D., Friston, K. J., Frackowiak, R. S., and Grasby, P. M. (1995). Dopaminergic modulation of impaired cognitive activation in the anterior cingulate cortex in schizophrenia. Nature 378, 180-182. doi: 10 . 1038/378180a0

Engell, A. D., and McCarthy, G. (2010). Selective attention modulates face-specific induced gamma oscillations recorded from ventral occipitotemporal cortex. J. Neurosci. 30, 8780-8786. doi: 10.1523/JNEUROSCI.1575-10.2010

Fan, J., Byrne, J., Worden, M. S., Guise, K. G., McCandliss, B. D., Fossella, J., et al. (2007). The relation of brain oscillations to attentional networks. J. Neurosci. 27, 6197-6206. doi: 10.1523/jneurosci.1833-07.2007

Fletcher, P. C., Frith, C. D., Grasby, P. M., Friston, K. J., and Dolan, R. J. (1996). Local and distributed effects of apomorphine on fronto-temporal function in acute unmedicated schizophrenia. J. Neurosci. 16, 7055-7062.

Floresco, S. B., Magyar, O., Ghods-Sharifi, S., Vexelman, C., and Tse, M. T. (2006). Multiple dopamine receptor subtypes in the medial prefrontal cortex of the rat regulate set-shifting. Neuropsychopharmacology 31, 297-309. doi: 10.1038/sj. npp. 1300825

Fornito, A., Yucel, M., Dean, B., Wood, S. J., and Pantelis, C. (2009). Anatomical abnormalities of the anterior cingulate cortex in schizophrenia: bridging the gap between neuroimaging and neuropathology. Schizophr. Bull. 35, 973-993. doi: 10.1093/schbul/sbn025

Fountoulakis, K. N., Giannakopoulos, P., Kovari, E., and Bouras, C. (2008). Assessing the role of cingulate cortex in bipolar disorder: neuropathological, structural 
and functional imaging data. Brain Res. Rev. 59, 9-21. doi: 10.1016/j.brainresrev. 2008.04.005

Franklin, B. J., and Paxinos, G. (2008). The Mouse Brain in Stereotaxic Coordinates. New York: Academic Press.

Fries, P., Nikolic, D., and Singer, W. (2007). The gamma cycle. Trends Neurosci. 30, 309-316. doi: 10.1016/j.tins.2007.05.005

Frodl, T., and Skokauskas, N. (2012). Meta-analysis of structural MRI studies in children and adults with attention deficit hyperactivity disorder indicates treatment effects. Acta Psychiatr. Scand. 125, 114-126. doi: 10.1111/j.1600-0447. 2011.01786.x

Furth, K. E., Mastwal, S., Wang, K. H., Buonanno, A., and Vullhorst, D. (2013). Dopamine, cognitive function and gamma oscillations: role of D4 receptors. Front. Cell. Neurosci. 7:102. doi: 10.3389/fncel.2013.00102

Gandal, M. J., Edgar, J. C., Klook, K., and Siegel, S. J. (2012). Gamma synchrony: towards a translational biomarker for the treatment-resistant symptoms of schizophrenia. Neuropharmacology 62, 1504-1518. doi: 10.1016/j.neuropharm. 2011.02.007

Gee, S., Ellwood, I., Patel, T., Luongo, F., Deisseroth, K., and Sohal, V. S. (2012). Synaptic activity unmasks dopamine D2 receptor modulation of a specific class of layer V pyramidal neurons in prefrontal cortex. J. Neurosci. 32, 4959-4971. doi: 10.1523/JNEUROSCI.5835-11.2012

Glykos, V., Whittington, M. A., Kaiser, M., and Lebeau, F. E. N. (2012). Catecholamine modulation of network oscillations in the rat medial prefrontal cortex in vitro. Soc. Neurosci. Abs. 2012:646.13. Available online at: http://am2012.sfn.org/am2012/.

Gorelova, N., Seamans, J. K., and Yang, C. R. (2002). Mechanisms of dopamine activation of fast-spiking interneurons that exert inhibition in rat prefrontal cortex. J. Neurophysiol. 88, 3150-3166. doi: 10.1152/jn.00335.2002

Gurden, H., Takita, M., and Jay, T. M. (2000). Essential role of D1 but not D2 receptors in the NMDA receptor-dependent long-term potentiation at hippocampalprefrontal cortex synapses in vivo. J. Neurosci. 20:RC106.

Gurevicius, K., Gureviciene, I., Valjakka, A., Schachner, M., and Tanila, H. (2004). Enhanced cortical and hippocampal neuronal excitability in mice deficient in the extracellular matrix glycoprotein tenascin-R. Mol. Cell. Neurosci. 25, 515523. doi: 10.1016/j.mcn.2003.12.001

Howard, M. W., Rizzuto, D. S., Caplan, J. B., Madsen, J. R., Lisman, J., Aschenbrenner-Scheibe, R., et al. (2003). Gamma oscillations correlate with working memory load in humans. Cereb. Cortex 13, 1369-1374. doi: 10. 1093/cercor/bhg084

Ko, J. H., Ptito, A., Monchi, O., Cho, S. S., Van Eimeren, T., Pellecchia, G., et al. (2009). Increased dopamine release in the right anterior cingulate cortex during the performance of a sorting task: a [11C]FLB 457 PET study. Neuroimage 46 , 516-521. doi: 10.1016/j.neuroimage.2009.02.031

Kocsis, B., Lee, P., and Deth, R. (2013). Enhancement of gamma activity after selective activationof dopamine D4 receptors in freely moving rats and in a neurodevelopmental model of schizophrenia. Brain Struct. Funct. doi: 10. 1007/s00429-013-0607-6. [Epub ahead of print].

Kömek, K., Bard Ermentrout, G., Walker, C. P., and Cho, R. Y. (2012). Dopamine and gamma band synchrony in schizophrenia-insights from computational and empirical studies. Eur. J. Neurosci. 36, 2146-2155. doi: 10.1111/j.1460-9568. 2012.08071.x

Kroener, S., Chandler, L. J., Phillips, P. E., and Seamans, J. K. (2009). Dopamine modulates persistent synaptic activity and enhances the signal-to-noise ratio in the prefrontal cortex. PLoS One 4:e6507. doi: 10.1371/journal.pone.0006507

Kuznetsova, A. Y., and Deth, R. C. (2008). A model for modulation of neuronal synchronization by D4 dopamine receptor-mediated phospholipid methylation. J. Comput. Neurosci. 24, 314-329. doi: 10.1007/s10827-007-0057-3

Kwok, J. C., Dick, G., Wang, D., and Fawcett, J. W. (2011). Extracellular matrix and perineuronal nets in CNS repair. Dev. Neurobiol. 71, 1073-1089. doi: 10. 1002/dneu.20974

Kyriakopoulos, M., Dima, D., Roiser, J. P., Corrigall, R., Barker, G. J., and Frangou, S. (2012). Abnormal functional activation and connectivity in the working memory network in early-onset schizophrenia. J. Am. Acad. Child Adolesc. Psychiatry 51, 911-920.e2. doi: 10.1016/j.jaac.2012.06.020

Lataster, J., Collip, D., Ceccarini, J., Haas, D., Booij, L., van Os, J., et al. (2011). Psychosocial stress is associated with in vivo dopamine release in human ventromedial prefrontal cortex: a positron emission tomography study using $\left[{ }^{18}\right.$ F]fallypride. Neuroimage 58, 1081-1089. doi: 10.1016/j.neuroimage.2011. 07.030
Lauzon, N. M., Bishop, S. F., and Laviolette, S. R. (2009). Dopamine D1 versus D4 receptors differentially modulate the encoding of salient versus nonsalient emotional information in the medial prefrontal cortex. J. Neurosci. 29, 48364845. doi: 10.1523/JNEUROSCI.0178-09.2009

Lewis, D. A., Fish, K. N., Arion, D., and Gonzalez-Burgos, G. (2011). Perisomatic inhibition and cortical circuit dysfunction in schizophrenia. Curr. Opin. Neurobiol. 21, 866-872. doi: 10.1016/j.conb.2011.05.013

Lumme, V., Aalto, S., Ilonen, T., Nagren, K., and Hietala, J. (2007). Dopamine D2/D3 receptor binding in the anterior cingulate cortex and executive functioning. Psychiatry Res. 156, 69-74. doi: 10.1016/j.pscychresns.2006. 12.012

Mauney, S. A., Athanas, K. M., Pantazopoulos, H., Shaskan, N., Passeri, E., Berretta, S., et al. (2013). Developmental pattern of perineuronal nets in the human prefrontal cortex and their deficit in schizophrenia. Biol. Psychiatry 74, 427-435. doi: 10.1016/j.biopsych.2013.05.007

Minzenberg, M. J., Laird, A. R., Thelen, S., Carter, C. S., and Glahn, D. C. (2009). Meta-analysis of 41 functional neuroimaging studies of executive function in schizophrenia. Arch. Gen. Psychiatry 66, 811-822. doi: 10.1001/ archgenpsychiatry.2009.91

Mulert, C., Leicht, G., Hepp, P., Kirsch, V., Karch, S., Pogarell, O., et al. (2010). Single-trial coupling of the gamma-band response and the corresponding BOLD signal. Neuroimage 49, 2238-2247. doi: 10.1016/j.neuroimage.2009. 10.058

Niessing, J., Ebisch, B., Schmidt, K. E., Niessing, M., Singer, W., and Galuske, R. A. (2005). Hemodynamic signals correlate tightly with synchronized gamma oscillations. Science 309, 948-951. doi: 10.1126/science.1110948

Onn, S. P., and Grace, A. A. (1994). Dye coupling between rat striatal neurons recorded in vivo: compartmental organization and modulation by dopamine. J. Neurophysiol. 71, 1917-1934.

Onn, S. P., Wang, X. B., Lin, M., and Grace, A. A. (2006). Dopamine D1 and D4 receptor subtypes differentially modulate recurrent excitatory synapses in prefrontal cortical pyramidal neurons. Neuropsychopharmacology 31, 318-338. doi: 10.1038/sj.npp.1300829

Rivera, A., Penafiel, A., Megias, M., Agnati, L. F., Lopez-Tellez, J. F., Gago, B., et al. (2008). Cellular localization and distribution of dopamine $\mathrm{D}(4)$ receptors in the rat cerebral cortex and their relationship with the cortical dopaminergic and noradrenergic nerve terminal networks. Neuroscience 155, 997-1010. doi: 10. 1016/j.neuroscience.2008.05.060

Roopun, A. K., Lebeau, F. E., Rammell, J., Cunningham, M. O., Traub, R. D., and Whittington, M. A. (2010). Cholinergic neuromodulation controls directed temporal communication in neocortex in vitro. Front. Neural Circuits 4:8. doi: 10.3389/fncir.2010.00008

Roopun, A. K., Middleton, S. J., Cunningham, M. O., LeBeau, F. E., Bibbig, A., Whittington, M. A., et al. (2006). A beta2-frequency $(20-30 \mathrm{~Hz})$ oscillation in nonsynaptic networks of somatosensory cortex. Proc. Natl. Acad. Sci. U S A 103, 15646-15650. doi: 10.1073/pnas.0607443103

Rushworth, M. F., Behrens, T. E., Rudebeck, P. H., and Walton, M. E. (2007). Contrasting roles for cingulate and orbitofrontal cortex in decisions and social behaviour. Trends Cogn. Sci. 11, 168-176. doi: 10.1016/j.tics.2007.01.004

Scheeringa, R., Fries, P., Petersson, K. M., Oostenveld, R., Grothe, I., Norris, D. G., et al. (2011). Neuronal dynamics underlying high- and low-frequency EEG oscillations contribute independently to the human BOLD signal. Neuron 69, 572-583. doi: 10.1016/j.neuron.2010.11.044

Schweimer, J., and Hauber, W. (2006). Dopamine D1 receptors in the anterior cingulate cortex regulate effort-based decision making. Learn. Mem. 13, 777782. doi: 10.1101/lm.409306

Seamans, J. K., and Yang, C. R. (2004). The principal features and mechanisms of dopamine modulation in the prefrontal cortex. Prog. Neurobiol. 74, 1-58. doi: 10.1016/j.pneurobio.2004.05.006

Simpson, E. H., Kellendonk, C., and Kandel, E. (2010). A possible role for the striatum in the pathogenesis of the cognitive symptoms of schizophrenia. Neuron 65, 585-596. doi: 10.1016/j.neuron.2010.02.014

Sohal, V. S., Zhang, F., Yizhar, O., and Deisseroth, K. (2009). Parvalbumin neurons and gamma rhythms enhance cortical circuit performance. Nature 459, 698702. doi: 10.1038/nature07991

Steullet, P., Cabungcal, J. H., Kulak, A., Kraftsik, R., Chen, Y., Dalton, T. P., et al. (2010). Redox dysregulation affects the ventral but not dorsal hippocampus: impairment of parvalbumin neurons, gamma oscillations and related behaviors. J. Neurosci. 30, 2547-2558. doi: 10.1523/JNEUROSCI.3857-09.2010 
St Onge, J. R., Abhari, H., and Floresco, S. B. (2011). Dissociable contributions by prefrontal D1 and D2 receptors to risk-based decision making. J. Neurosci. 31, 8625-8633. doi: 10.1523/JNEUROSCI.1020-11.2011

Sugiyama, S., Prochiantz, A., and Hensch, T. K. (2009). From brain formation to plasticity: insights on Otx2 homeoprotein. Dev. Growth Differ. 51, 369-377. doi: 10.1111/j.1440-169x.2009.01093.X

Suhara, T., Okubo, Y., Yasuno, F., Sudo, Y., Inoue, M., Ichimiya, T., et al. (2002). Decreased dopamine D2 receptor binding in the anterior cingulate cortex in schizophrenia. Arch. Gen. Psychiatry 59, 25-30. doi: 10.1001/archpsyc.59.1.25

Thurley, K., Senn, W., and Luscher, H. R. (2008). Dopamine increases the gain of the input-output response of rat prefrontal pyramidal neurons. J. Neurophysiol. 99, 2985-2997. doi: 10.1152/jn.01098.2007

Tierney, P. L., Thierry, A. M., Glowinski, J., Deniau, J. M., and Gioanni, Y. (2008). Dopamine modulates temporal dynamics of feedforward inhibition in rat prefrontal cortex in vivo. Cereb. Cortex 18, 2251-2262. doi: 10.1093/cercor/ bhm 252

Towers, S. K., and Hestrin, S. (2008). D1-like dopamine receptor activation modulates GABAergic inhibition but not electrical coupling between neocortical fast-spiking interneurons. J. Neurosci. 28, 2633-2641. doi: 10.1523/JNEUROSCI. 5079-07.2008

Tritsch, N. X., and Sabatini, B. L. (2012). Dopaminergic modulation of synaptic transmission in cortex and striatum. Neuron 76, 33-50. doi: 10.1016/j.neuron. 2012.09.023

Tseng, K. Y., and O'Donnell, P. (2007). Dopamine modulation of prefrontal cortical interneurons changes during adolescence. Cereb. Cortex 17, 1235-1240. doi: 10. 1093/cercor/bhl034

Vijayraghavan, S., Wang, M., Birnbaum, S. G., Williams, G. V., and Arnsten, A. F. (2007). Inverted-U dopamine D1 receptor actions on prefrontal neurons engaged in working memory. Nat. Neurosci. 10, 376-384. doi: 10.1038/nn1846

Vogt, B. A., and Paxinos, G. (2014). Cytoarchitecture of mouse and rat cingulate cortex with human homologies. Brain Struct. Funct. 219, 185-192. doi: 10. 1007/s00429-012-0493-3

Weiss, T., Veh, R. W., and Heinemann, U. (2003). Dopamine depresses cholinergic oscillatory network activity in rat hippocampus. Eur. J. Neurosci. 18, 2573-2580. doi: 10.1046/j.1460-9568.2003.02970.x
Whittington, M. A., Roopun, A. K., Traub, R. D., and Davies, C. H. (2011). Circuits and brain rhythms in schizophrenia: a wealth of convergent targets. Curr. Opin. Pharmacol. 11, 508-514. doi: 10.1016/j.coph.2011.04.010

Wójtowicz, A. M., van den Boom, L., Chakrabarty, A., Maggio, N., Haq, R. U., Behrens, C. J., et al. (2009). Monoamines block kainate- and carbachol-induced gamma-oscillations but augment stimulus-induced gamma-oscillations in rat hippocampus in vitro. Hippocampus 19, 273-288. doi: 10.1002/hipo.20508

Woo, T. U., Kim, A. M., and Viscidi, E. (2008). Disease-specific alterations in glutamatergic neurotransmission on inhibitory interneurons in the prefrontal cortex in schizophrenia. Brain Res. 1218, 267-277. doi: 10.1016/j.brainres.2008. 03.092

Yamawaki, N., Stanford, I. M., Hall, S. D., and Woodhall, G. L. (2008). Pharmacologically induced and stimulus evoked rhythmic neuronal oscillatory activity in the primary motor cortex in vitro. Neuroscience 151, 386-395. doi: 10.1016/j. neuroscience.2007.10.021

Zhang, K., Grady, C. J., Tsapakis, E. M., Andersen, S. L., Tarazi, F. I., and Baldessarini, R. J. (2004). Regulation of working memory by dopamine D4 receptor in rats. Neuropsychopharmacology 29, 1648-1655. doi: 10.1038/sj.npp. 1300491

Conflict of Interest Statement: The authors declare that the research was conducted in the absence of any commercial or financial relationships that could be construed as a potential conflict of interest.

Received: 29 July 2013; accepted: 01 August 2014; published online: 20 August 2014. Citation: Steullet P, Cabungcal J-H, Cuénod M and Do KQ (2014) Fast oscillatory activity in the anterior cingulate cortex: dopaminergic modulation and effect of perineuronal net loss. Front. Cell. Neurosci. 8:244. doi: 10.3389/fncel.2014.00244 This article was submitted to the journal Frontiers in Cellular Neuroscience. Copyright (c) 2014 Steullet, Cabungcal, Cuénod and Do. This is an open-access article distributed under the terms of the Creative Commons Attribution License (CC BY). The use, distribution or reproduction in other forums is permitted, provided the original author(s) or licensor are credited and that the original publication in this journal is cited, in accordance with accepted academic practice. No use, distribution or reproduction is permitted which does not comply with these terms. 